\title{
Nomograms to predict the presence and extent of inguinal lymph node metastasis in penile cancer patients with clinically positive lymph nodes
}

\author{
Xiang Zhou ${ }^{1 \#}$, Yan Zhong ${ }^{2 \#}$, Lebin Song ${ }^{3 \#}$, Yamin Wang ${ }^{1}$, Yichun Wang ${ }^{1}$, Qijie Zhang ${ }^{1}$, Rong Cong ${ }^{1}$, \\ Chengjian Ji ${ }^{1}$, Tongfu Yu ${ }^{2}$, Ninghong Song ${ }^{1}$
}

${ }^{1}$ Department of Urology, First Affiliated Hospital of Nanjing Medical University, Nanjing 210029, China; ${ }^{2}$ Department of Radiology, The First Affiliated Hospital of Nanjing Medical University, Nanjing 210029, China; ${ }^{3}$ Department of Dermatology, the First Affiliated Hospital of Nanjing Medical University, Nanjing 210029, China

Contributions: (I) Conception and design: T Yu, N Song; (II) Administrative support: T Yu, N Song; (III) Provision of study materials or patients: X Zhou, Y Zhong; (IV) Collection and assembly of data: X Zhou, Y Zhong, L Song, Q Zhang, R Cong, C Ji; (V) Data analysis and interpretation: X Zhou, Y Zhong, L Song; (VI) Manuscript writing: All authors; (VII) Final approval of manuscript: All authors.

\#These authors contributed equally to this work.

Correspondence to: Ninghong Song, MD. Department of Urology, the First Affiliated Hospital of Nanjing Medical University, No. 300, Guangzhou Street, Nanjing 210029, China. Email: songninghong@126.com; Tongfu Yu, MD. Department of Radiology, The First Affiliated Hospital of Nanjing Medical University, No. 300, Guangzhou Street, Nanjing 210029, China. Email: yu.tongfu@163.com.

Background: We developed two nomograms to predict the presence of inguinal lymph node metastasis (ILNM) and two or more histologically positive inguinal lymph nodes (ILNs) or one ILN with extranodal extension (ENE) in penile cancer patients with clinically positive inguinal lymph nodes (ILNs).

Methods: This study enrolled 75 penile squamous cell carcinoma (PSCC) patients with clinically positive ILNs. A binary logistic regression model was taken to screen out the risk factors for the presence of ILNM and two or more positive ILNs or one node with ENE in one side. Nomograms were plotted based on the results of multivariate logistic regression analysis.

Results: Our multivariate analysis demonstrated that the pathological grade, lymphovascular invasion (LVI), and short diameter (SD) of the largest ILN were independent risks. The bootstrap-corrected concordance index of the nomogram for the presence of ILNM was 0.948 , and 0.878 for two or more histologically positive ILNs or one ILN with ENE on one side.

Conclusions: By incorporating tumor pathological grade, LVI and SD of the largest ILN, our nomograms can efficiently predict the ILNM in penile cancer patients with clinically positive nodes.

Keywords: Penile cancer; lymph node metastasis (LNM); clinically positive lymph nodes; lymph node dissection; nomogram

Submitted Aug 19, 2019. Accepted for publication Jan 05, 2020.

doi: $10.21037 /$ tau.2020.01.32

View this article at: http://dx.doi.org/10.21037/tau.2020.01.32

\section{Introduction}

Penile carcinoma (PC), a rare malignancy, accounts for less than $0.1 \%$ of all male neoplasms in the developed countries. In 2019, for example, about 2,000 cases of PC were diagnosed in America (1). However, this incidence rises to $10-20 \%$ in some developing countries, such as
Brazil and Uganda (2).The presence and extent of lymph node metastasis (LNM) were the high-value prognostic factors for PC (3). Clinically positive lymph node (LN) is a significant indictor for LNM, and $40-70 \%$ of the patients with positive LNs develop metastatic lymphadenopathy finally. The enlarged LNs in other cases are often benign 
because of the local inflammatory changes (4-6). Nowadays, radical inguinal lymph node dissection (ILND) is the most optimal method for identifying the aetiology of enlarged inguinal lymph nodes (ILNs) and the metastatic potential of the remaining non-enlarged nodes (3). But for patients with clinically positive nodes, an alternative procedure is fine needle aspiration cytology (FNAC) under the guidance of ultrasound (US) (6). Even though, about 30-50\% of patients with palpable ILNs were overtreatment based on guidelines for treatments of PC patients with palpable nodes $(4,7)$. Apart from this, approximately $40-70 \%$ of patients treated by radical ILND suffer from surgery-aroused complications, either minor $(65.7 \%)$ or major complications (34.3\%) (7).

According to European Association of Urology (EAU) and National Comprehensive Cancer Network (NCCN) guidelines on penile cancer, when two or more positive ILNs or one node with extranodal extension (ENE) are found on one side, pelvic lymph node dissection (PLND) can be considered and performed during or after ILND according to the intraoperative frozen section results or the pathologic results of ILND-collected nodes $(3,8)$. However, this procedure is expensive and consumes too much time and money in waiting for the results of intraoperative frozen section or reoperation. Therefore, we developed two nomograms to predict the presence of inguinal lymph nodes metastasis (ILNM) and two or more positive ILNs or one node with ENE in one side.

\section{Methods}

This retrospective study included 236 patients with penile squamous cell carcinoma (PSCC), who were treated in our centre between January 2010 and March 2019. Criteria of eligibility: (I) clinically positive ILNs were present in patients; (II) enhanced computed tomography (CT) or enhanced magnetic resonance imaging (MRI) had been used to evaluate the ILNs of PC patients before surgery in our centre; (III) patients had undergone ILND and their dissected lymph nodes had been pathologically staged when the primary lesion was removed. This study was approved by ethics committee of the First Affiliated Hospital of Nanjing Medical University.

\section{Patbological evaluation}

The pathological results of original cancer slides were retrieved. And, the 2017 TNM staging system was applied for tumor staging. And, well differentiated (G1), moderately differentiated (G2), or poorly differentiated/undifferentiated (G3-4) tumor relied mainly on Broder's histopathologic grading (9). G1 and G2 were considered as low grade, G3 and G4 as high grade, T3-4 as high stage, and Tis, Ta, T1 and T2 as low stage. Tumor clusters walled in endothelium lined spaces was identified as lymphovascular invasion (LVI).

\section{Imaging evaluation}

Original imaging results of patients before surgery were retrieved. All ILNs $\geq 3 \mathrm{~mm}$ in size were counted totally in bilateral inguinal regions. ILNs less than $3 \mathrm{~mm}$ were excluded because they were likely derived from vascular structures or other nonspecific soft tissues. In addition, the short diameter (SD) of the largest ILN was measured. The internal heterogeneity and border morphology of the largest ILN were examined.

\section{Statistical analysis}

SPSS 23.0 (IBM, Armonk, New York, USA) and R version 3.6.0 (The R Foundation for Statistical Computing, Vienna, Austria) were applied for statistical analyses and graphics. Continuous measurements about clinical features were represented as mean (range). Student's T test, MannWhitney $U$ and $\chi^{2}$ test were conducted for continuous, ordered categorical and categorical variables respectively. A binary logistic regression model was constructed to evaluate the risk factors for the presence of ILNM and two or more positive ILNs or one node with ENE in one side. Nomograms were developed based on the results of multivariate logistic regression analysis. Receiver operating characteristic curve (ROC) was used to graphically show the predictive accuracy of the models, which was quantified by the areas under the curves (AUC). The two nomograms were caliberated and internally validated by performing bootstraps with 1,000 resamples. Calibration curves were plotted to evaluated the calibration of the models. $\mathrm{P}$ value less than 0.05 was considered significant.

\section{Results}

\section{Detailed characteristics}

A total of 75 PSCC patients (mean age 55.9 years; range, 25 to 79 years) were eligible, including 5 (6.7\%) in Tis, $2(2.7 \%)$ in $\mathrm{Ta}, 28(37.3 \%)$ in $\mathrm{T} 1,21(28.0 \%)$ in $\mathrm{T} 2,17$ 
Table 1 Basic characteristics of patients cohort

\begin{tabular}{|c|c|}
\hline Characteristics & Value \\
\hline Mean age, yr (range) & $55.9(25-79)$ \\
\hline \multicolumn{2}{|l|}{ Histopathological evaluation } \\
\hline \multicolumn{2}{|l|}{ Pathological T stage, n (\%) } \\
\hline Tis & $5(6.7)$ \\
\hline $\mathrm{Ta}$ & $2(2.7)$ \\
\hline $\mathrm{T} 1$ & $28(37.3)$ \\
\hline $\mathrm{T} 2$ & $21(28.0)$ \\
\hline T3 & $17(22.6)$ \\
\hline $\mathrm{T} 4$ & $2(2.7)$ \\
\hline \multicolumn{2}{|l|}{ Grade, n (\%) } \\
\hline 1 & $20(26.6)$ \\
\hline 2 & $29(38.7)$ \\
\hline $3-4$ & $26(34.7)$ \\
\hline \multicolumn{2}{|l|}{ Lymphovascular invasion, n (\%) } \\
\hline Absent & $36(48)$ \\
\hline Present & $39(52)$ \\
\hline \multicolumn{2}{|l|}{ Imaging evaluation } \\
\hline Mean detected nodes $\geq 3 \mathrm{~mm}$ (range) & $10.9(2-28)$ \\
\hline SD of the largest node (range) & $14.9(4.1-56.9)$ \\
\hline \multicolumn{2}{|l|}{ Shape of the largest node, $\mathrm{n}(\%)$} \\
\hline Oval & $53(70.7)$ \\
\hline Round/irregular & $22(29.3)$ \\
\hline \multicolumn{2}{|l|}{ Signal heterogeneity, n (\%) } \\
\hline Mixed & 25 (33.3) \\
\hline Homogeneous & $50(66.7)$ \\
\hline \multicolumn{2}{|l|}{ Border of the largest node, $\mathrm{n}(\%)$} \\
\hline Smooth & $56(74.7)$ \\
\hline Irregular & $19(25.3)$ \\
\hline \multicolumn{2}{|l|}{ ILNM, n (\%) } \\
\hline Absent & $44(58.7)$ \\
\hline Present & $31(41.3)$ \\
\hline \multicolumn{2}{|l|}{ ILNM ( $\geq 2)$ or ENE, n (\%) } \\
\hline Absent & $50(66.7)$ \\
\hline Present & 25 (33.3) \\
\hline
\end{tabular}

$\mathrm{SD}$, short diameter; ILNM, inguinal lymph node metastasis; $\operatorname{ILNM}(\geq 2)$ or ENE, two or more positive ILNs or one node with extranodal extension in one side.
$(22.6 \%)$ in $\mathrm{T} 3$, and $2(2.7 \%)$ in $\mathrm{T} 4$ tumor stage. The tumors were mainly in G2 (38.7\%) and G3-4 (34.7\%). LVI was detected in $39(52 \%)$ patients, and the rest were LVI-negative. As to imaging results, the mean number of palpable ILNs $\geq 3 \mathrm{~mm}$ in size in bilateral inguinal regions was 10.9 (range, 2-28). And, the mean SD of the largest ILN was $14.9 \mathrm{~mm}$ (range, 4.1-56.9 mm). The largest ILN showed internal heterogeneity in 25 (33.3\%), an irregular border in $19(25.3 \%)$, and a round/irregular shape in 22 $(29.3 \%)$ patients. Of 31 cases undertaking ILNM, 25 showed two or more positive ILNs or one node with ENE in one side. The detailed clinicopathological characteristics of eligible patients were shown in Table 1.

\section{Nomogram for the presence of ILNM}

Our multivariate analysis demonstrated that tumor grade [G3-4 vs. G1-2: odds ratio (OR) 6.467; 95\% confidence interval (CI), 1.241-33.684], LVI (OR 5.162, 95\% CI 1.05625.243), and SD of the largest ILN (OR 1.349; 95\% CI, 1.133-1.606 ) were independent predictors of the presence of ILNM in PC cases (Table 2). Age, tumor stage, total number of $\geq 3 \mathrm{~mm}$ ILNs, border shape and internal heterogeneity of the largest node were not significant predictors of ILNM (Table 2). Subsequently, a nomogram was developed to predict the metastasis of ILNs (Figure 1). The bootstrap-corrected concordance index of this nomogram was 0.948 (Figure $2 A$ ). The calibration plots demonstrated that its predictive accuracy was good (Figure S1A).

\section{Nomogram for two or more positive ILNs or one ILN with ENE on one side}

According to the multivariate logistic regression analysis, tumor grade (G3-4 vs. G1-2: OR 4.431; 95\% CI, $1.303-$ 15.070), LVI (OR 4.514; 95\% CI, 1.156-17.620), and SD of the largest ILN (OR 1.070; 95\% CI, 1.002-1.142) were related to the occurrence of two or more positive ILNs or one ILN with ENE (Table 3). Therefore, a nomogram was developed to predict the occurrence of two or more positive ILNs or one ILN with ENE, the bootstrap corrected concordance index of which was 0.878 (Figure 2B), and the nomogram was shown in Figure 3. The calibration plots suggested that the accuracy was good (Figure S1B).

\section{Discussion}

Radical ILND can bring satisfactory outcome to $80 \%$ of the 
Table 2 Univariate and multivariate analysis of clinicopathologic and imaging factors to predict inguinal lymph node involvement

\begin{tabular}{lccc}
\hline \multirow{2}{*}{ Variable } & Univariate analysis & & Multivariate analysis \\
\cline { 2 - 3 } & OR $(95 \% \mathrm{Cl})$ & $\mathrm{P}$ & $\mathrm{OR}(95 \% \mathrm{Cl})$ \\
\hline Age & $1.007(0.969-1.046)$ & 0.725 & $\mathrm{P}$ \\
Grade (G3-4 vs. G1-2) & $8.369(2.831-24.741)$ & 0.000 & $6.467(1.241-33.684)$ \\
T stage (T3-4 vs. T1-2) & $3.111(0.533-18.172)$ & 0.208 & 0.027 \\
LVI (present vs. absent) & $12.400(3.904-39.382)$ & 0.000 & $5.162(1.056-25.243)$ \\
Total detected nodes $\geq 3$ mm & $1.029(0.935-1.131)$ & 0.560 & 0.043 \\
SD of the largest node (continue) & $1.385(1.183-1.621)$ & 0.000 & $1.349(1.133-1.606)$ \\
Shape (round/irregular vs. oval) & $3.706(1.306-10.513)$ & 0.014 & 0.001 \\
Signal heterogeneity (mixed vs. homogeneous) & $10.028(3.258-30.860)$ & 0.000 & 0.216 \\
Border (irregular vs. smooth) & $25.500(5.226-124.435)$ & 0.000 & 0.142 \\
\hline
\end{tabular}

OR, odds ratio; Cl, confidence interval; LVI, lymphovascular invasion; SD, short diameter.
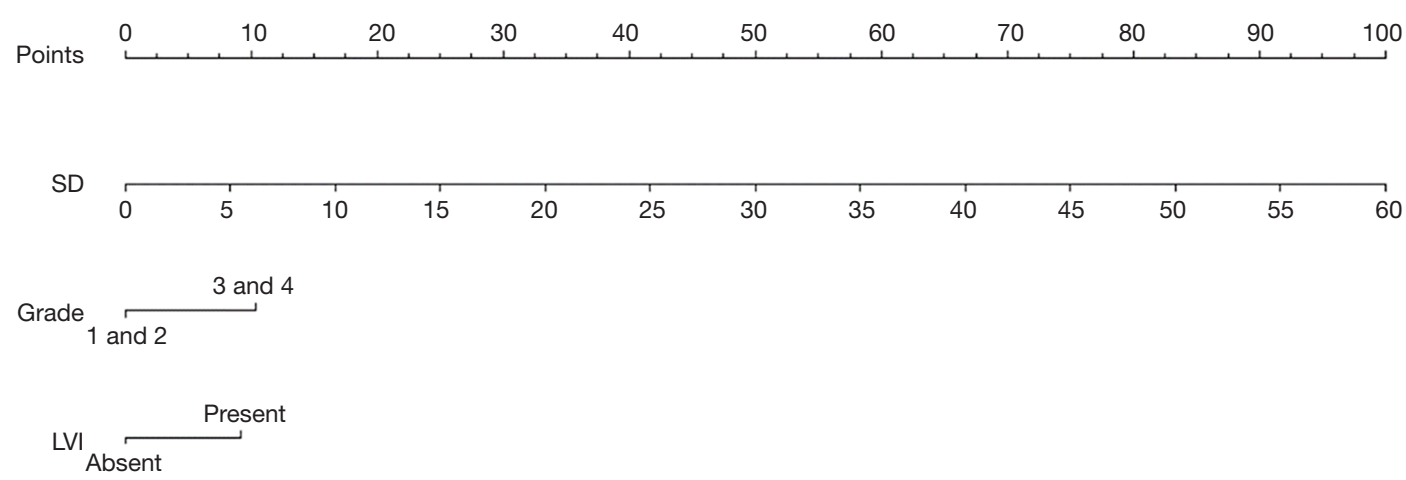

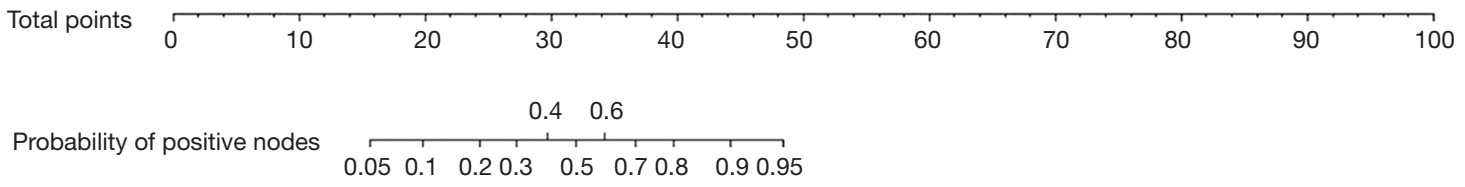

Figure 1 Nomogram predicting the presence of ILNM in patients with clinically positive ILNs. ILNM, inguinal lymph node metastasis; ILN, inguinal lymph node.

patients with low-volume histologically positive ILNs (10). However, approximately 40-70\% of patients still suffer from surgery-related complications, like skin necrosis (in $7-61 \%$ ), wound infection (in 7-40\%), seroma (in 5-30\%), lymphocele (in 2-10\%), and lymphedema (in 18-38\%) (7). Among them, the incidence of wound infections has shown a declining trend, but others still remain at a high level (11). As for minimally invasive surgery for ILND, a lower complication rate of about $30 \%$, but ILND-caused lymphocele reaches $12-27 \%$, higher than that of open ILND $(12,13)$. FNAC under the guidance of US can detect tumor in palpable nodes accurately, rapidly and easily $(3,6)$. But, its results are useable only when biopsy is pathologically positive and false-negative rate was reportedly up to $15 \%(14,15)$. Repetition of FNAC or surgical staging is necessary, once biopsy is tumor negative. Clinically positive nodes exist in 

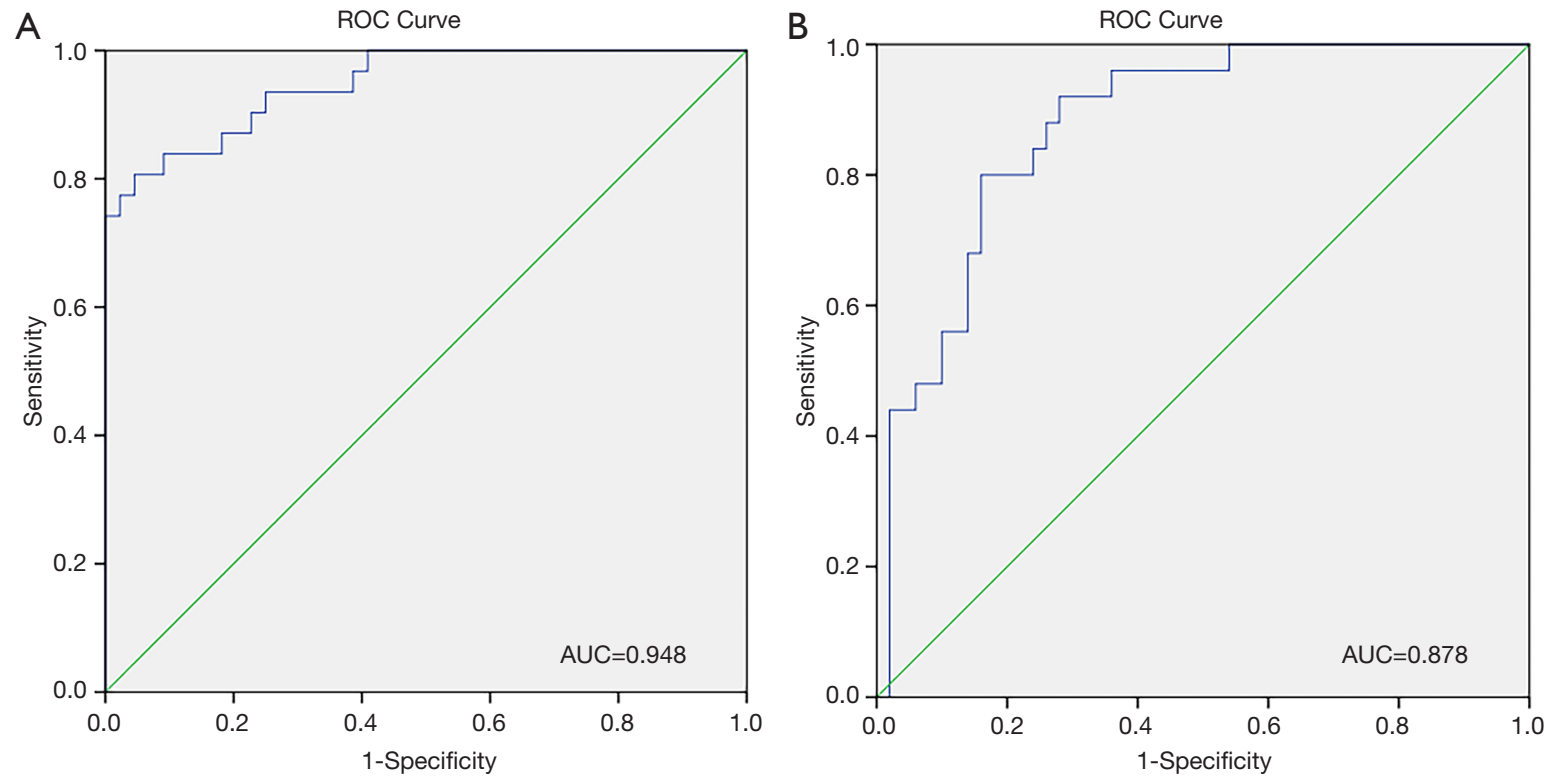

Figure 2 ROC curve demonstrating diagnostic performance of nomograms. (A) ROC for the nomogram predicting the presence of ILNM; (B) ROC for the nomogram predicting two or more histologically positive ILNs or one ILN with ENE on one side. ROC, receiver operating characteristic curve; AUC, area under the curve; ILNM, inguinal lymph node metastasis; ILN, inguinal lymph node; ENE, extranodal extension.

Table 3 Univariate and multivariate analysis of clinicopathologic and imaging factors to predict two or more histologically positive ILNs or one ILN with ENE in one side

\begin{tabular}{|c|c|c|c|c|}
\hline Variable & \multicolumn{2}{|c|}{ Univariate analysis } & \multicolumn{2}{|c|}{ Multivariate analysis } \\
\hline Age & $0.999(0.960-1.039)$ & 0.946 & & \\
\hline Grade (G3-4 vs. G1-2) & $7.111(2.437-20.753)$ & 0.000 & $4.431(1.303-15.070)$ & 0.017 \\
\hline T stage (T3-4 vs. T1-2) & $4.571(0.776-26.923)$ & 0.093 & & \\
\hline Total detected nodes $\geq 3 \mathrm{~mm}$ & $1.030(0.934-1.136)$ & 0.556 & & \\
\hline SD of the largest node & $1.116(1.045-1.192)$ & 0.001 & $1.070(1.002-1.142)$ & 0.044 \\
\hline Shape (round/irregular vs. oval) & $3.692(1.296-10.518)$ & 0.014 & & \\
\hline Signal heterogeneity (mixed vs. homogeneous) & $6.000(2.082-17.292)$ & 0.001 & & \\
\hline
\end{tabular}

ILN, inguinal lymph node; ENE, extranodal extension; OR, odds ratio; Cl, confidence interval; LVI, lymphovascular invasion; SD, short diameter.

$28-64 \%$ of PC patients (16). Approximately $30-60 \%$ of PC patients with palpable ILNs do not experience ILNM (4-6), consistent with our results that $58.7 \%$ of the cases were ILNs histologically negative. So, we need to develop nomograms for choosing more appropriate clinically node positive PC patients for invasive node staging.

Our nomograms suggested that tumor grade and LVI were associated with ILNM in PC patients with clinically positive nodes, which is supported by the researches of Peak et al. (17) and Bhagat et al. (18). Large size, round or irregular shape, 


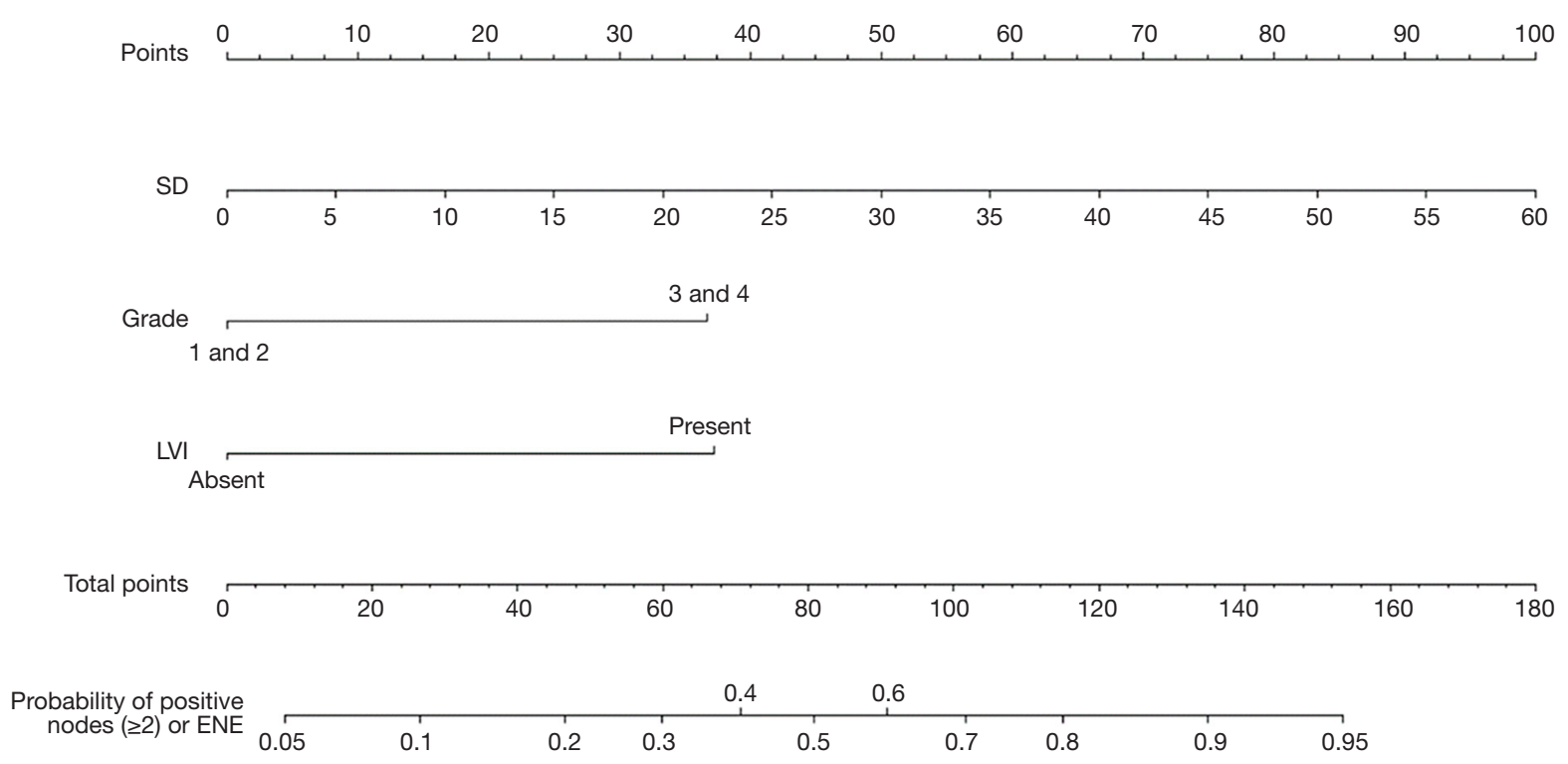

Figure 3 Nomogram predicting two or more histologically positive ILNs or one ILN with ENE on one side in patients with clinically positive ILNs. ILN, inguinal lymph node; ENE, extranodal extension.

heterogeneous signal and irregular border of the nodes were also associated with the tumor metastasis in LNs $(19,20)$. Therefore, we incorporated the imaging parameters of the largest ILN into the nomogram. Our multivariate logistic regression analysis showed that only SD of the largest ILN was an independent risk factor of ILNM, which is supported by the study of Tang et al. (19). Merely imaging examination by CT or MRI cannot detect tumor metastasis in palpable ILNs accurately, but imaging parameters combined with pathologic findings of primary tumor can predict ILNM more accurately. Compared with nomograms developed by Ficarra et al. (21), Peak et al. (17) and Bhagat et al. (18), ours is more specific to predict ILNs in PC patients with clinically positive ILNs, as shown by the bootstrapping-corrected concordance index (ours' $0.948 v s$. others' $0.876,0.880$ and 0.74 ).

Additionally, the rate of pelvic lymph node metastasis (PLNM) is $23 \%$ in patients with more than two histologically positive ILNs and $56 \%$ in those with more than three histologically positive ILNs, or one ILN with ENE. Ipsilateral PLND is recommended and can be performed during ILND when two or more positive ILNs or one ILN with ENE were diagnosed within intraoperative frozen section or later according to the pathologic results of ILND (3). The total number of ILNs collected through radical ILND is frequently more than 10 (11), so the frozen section preparation often prolongs the operating time. If PLND is preformed based on pathologic results of ILND, the patients always need reoperation and months for recovery. Our nomogram showed that tumor grade, LVI and SD of the largest ILN were the independent risk factors, with a bootstrapping-corrected concordance of 0.878. This nomogram can be applied to improve decisionmaking and to support treatment plans that PLND can be simultaneously performed during ILND, avoiding time wasting on waiting for the results of intraoperative frozen section and reoperation after ILND.

Some limitations exist in our study. The patients were collected from a single medical center. External validation is required. Furthermore, other pathological characteristics, such perineural invasion and pattern of invasion, were not analyzed, which may improve the bootstrapping-corrected concordance index of our nomograms if they were included. Nevertheless, this is an important first time in generating predictive nomograms for the presence of ILNM and two or more histologically positive ILNs or one ILN with ENE in patients with clinically positive ILNs.

\section{Conclusions}

It is the first time that nomograms are used to predict the 
presence of ILNM and two or more histologically positive ILNs or one ILN with ENE in patients with clinically positive ILNs. By incorporating tumor pathological grade, LVI and the SD of the largest ILN, our nomograms can efficiently predict the ILNM in PC patients with clinically positive nodes.

\section{Acknowledgments}

Funding: This work was supported by the Research Project of Jiangsu Provincial Commission of Health and Family Planning Commission (grant number Z201601).

\section{Footnote}

Conflicts of Interest: All authors have completed the ICMJE uniform disclosure form (available at http://dx.doi. org/10.21037/tau.2020.01.32). The authors have no conflicts of interest to declare.

Ethical Statement: The authors are accountable for all aspects of the work in ensuring that questions related to the accuracy or integrity of any part of the work are appropriately investigated and resolved. This study was approved by ethics committee of the First Affiliated Hospital of Nanjing Medical University (approval number: 2020-SR-005).

Open Access Statement: This is an Open Access article distributed in accordance with the Creative Commons Attribution-NonCommercial-NoDerivs 4.0 International License (CC BY-NC-ND 4.0), which permits the noncommercial replication and distribution of the article with the strict proviso that no changes or edits are made and the original work is properly cited (including links to both the formal publication through the relevant DOI and the license). See: https://creativecommons.org/licenses/by-ncnd/4.0\%.

\section{References}

1. Siegel RL, Miller KD, Jemal A. Cancer statistics, 2019. CA Cancer J Clin 2019;69:7-34.

2. Christodoulidou M, Sahdev V, Houssein S, et al. Epidemiology of penile cancer. Curr Probl Cancer 2015;39:126-36

3. Hakenberg OW, Compérat EM, Minhas S, et al. EAU guidelines on penile cancer: 2014 update. Eur Urol
2015;67:142-50.

4. Lont AP, Kroon BK, Gallee MP, et al. Pelvic lymph node dissection for penile carcinoma: extent of inguinal lymph node involvement as an indicator for pelvic lymph node involvement and survival. J Urol 2007;177:947.

5. Naumann CM, van der Horst $S$, van der Horst C, et al. Reliability of dynamic sentinel node biopsy combined with ultrasound-guided removal of sonographically suspicious lymph nodes as a diagnostic approach in patients with penile cancer with palpable inguinal lymph nodes. Urol Oncol 2015;33:389.e9-14.

6. Saisorn I, Lawrentschuk N, Leewansangtong S, et al. Fine-needle aspiration cytology predicts inguinal lymph node metastasis without antibiotic pretreatment in penile carcinoma. BJU Int 2006;97:1225-8.

7. Protzel C, Alcaraz A, Horenblas S, et al. Lymphadenectomy in the surgical management of penile cancer. Eur Urol 2009;55:1075.

8. Clark PE, Spiess PE, Agarwal N, et al. Penile cancer: Clinical Practice Guidelines in Oncology. J Natl Compr Canc Netw 2013;11:594-615.

9. Brierley, JD. TNM Classification of Malignant Tumours, 8th Edn. International Union Against Cancer, WileyBlackwell; 2016:188-9.

10. Leone A, Diorio GJ, Pettaway C, et al. Contemporary management of patients with penile cancer and lymph node metastasis. Nat Rev Urol 2017;14:335.

11. Gopman JM, Djajadiningrat RS, Baumgarten AS, et al. Predicting postoperative complications of inguinal lymph node dissection for penile cancer in an international multicentre cohort. BJU Int 2015;116:196-201.

12. Master VA, Jafri SM, Moses KA, et al. Minimally invasive inguinal lymphadenectomy via endoscopic groin dissection: comprehensive assessment of immediate and long-term complications. J Urol 2012;188:1176-80.

13. Kumar V, Sethia KK. Prospective study comparing video-endoscopic radical inguinal lymph node dissection (VEILND) with open radical ILND (OILND) for penile cancer over an 8-year period. BJU Int 2017;119:530-4.

14. Kulkarni JN, Kamat MR. Prophylactic bilateral groin node dissection versus prophylactic radiotherapy and surveillance in patients with $\mathrm{N} 0$ and N1-2A carcinoma of the penis. Eur Urol 1994;26:123-8

15. Horenblas S, Van Tinteren H, Delemarre JF, et al. Squamous cell carcinoma of the penis: accuracy of tumor, nodes and metastasis classification system, and role of lymphangiography, computerized tomography scan and fine needle aspiration cytology. J Urol 1991;146:1279-83. 
16. Ornellas AA, Seixas AL, Marota A, et al. Surgical treatment of invasive squamous cell carcinoma of the penis: retrospective analysis of 350 cases. J Urol 1994;151:1244-9.

17. Peak TC, Russell GB, Dutta R, et al. A National Cancer Database-based nomogram to predict lymph node metastasis in penile cancer. BJU Int 2019;123:1005-10.

18. Bhagat SK, Gopalakrishnan G, Kekre NS, et al. Factors predicting inguinal node metastasis in squamous cell cancer of penis. World J Urol 2010;28:93-8.

Cite this article as: Zhou X, Zhong Y, Song L, Wang Y, Wang Y, Zhang Q, Cong R, Ji C, Yu T, Song N. Nomograms to predict the presence and extent of inguinal lymph node metastasis in penile cancer patients with clinically positive lymph nodes. Transl Androl Urol 2020;9(2):621-628. doi: $10.21037 /$ tau.2020.01.32
19. Tang Y, Rao S, Yang C, et al. Value of MRI morphologic features with pT1-2 rectal cancer in determining lymph node metastasis. J Surg Oncol 2018;118:544-50.

20. Kochhar R, Taylor B, Sangar V. Imaging in primary penile cancer: current status and future directions. Eur Radiol 2010;20:36-47.

21. Ficarra V, Zattoni F, Artibani W, et al. Nomogram predictive of pathological inguinal lymph node involvement in patients with squamous cell carcinoma of the penis. J Urol 2006;175:1700-4. 


\section{Supplementary}
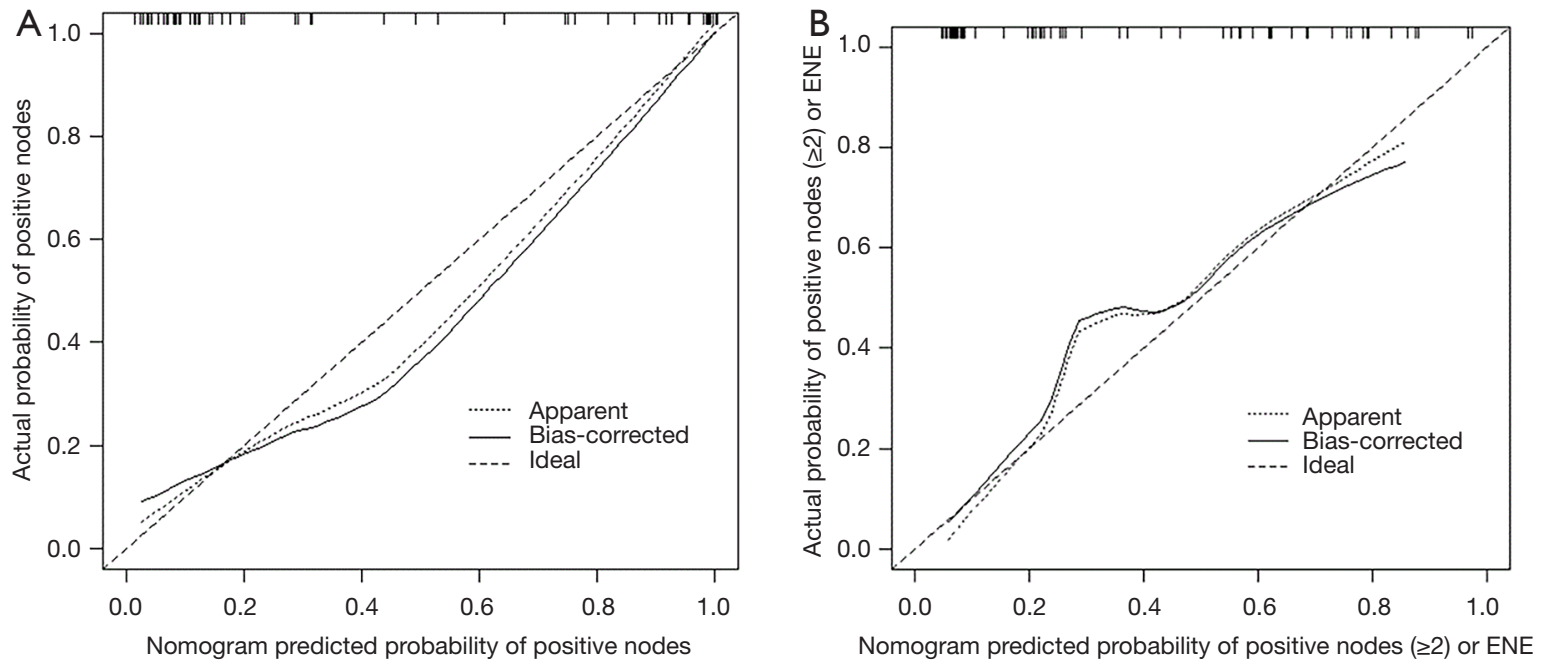

Figure S1 Calibration curve for two nomograms. (A) Nomogram predicting the presence of ILNM; (B) nomogram predicting two or more histologically positive ILNs or one ILN with ENE on one side. ILNM, inguinal lymph node metastasis; ILN, inguinal lymph node; ENE, extranodal extension. 